\title{
Identifying the MHD signature and power deposition characteristics associated with type-II ELMs in ASDEX Upgrade
}

\author{
C P Perez ${ }^{1,2}$, M Maraschek ${ }^{2}$, S da Graça ${ }^{3}, \mathbf{R}$ J Buttery ${ }^{1}$, A \\ Herrmann ${ }^{2}$, J Stober ${ }^{2}$, G Conway ${ }^{2}$, T Eich ${ }^{2}$, J C Fuchs ${ }^{2}$, L D \\ Horton $^{2}$, V Igochine ${ }^{2}$, A Kallenbach ${ }^{2}$, A Loarte ${ }^{4}$, H W Müller ${ }^{2}$, \\ I Nunes ${ }^{3}$, G Saibene ${ }^{4}$, R Sartori ${ }^{4}$, A C C Sips ${ }^{2}$, W Suttrop ${ }^{2}$, E \\ Wolfrum $^{2}$ and the ASDEX Upgrade Team \\ ${ }^{1}$ EURATOM/UKAEA Fusion Association, Culham Science Centre, Abingdon, OX14 \\ 3DB, UK \\ ${ }^{2}$ Max-Planck-Institut für Plasmaphysik, EURATOM-Association IPP, Garching, \\ D-85748, Germany \\ ${ }^{3}$ Associação EURATOM/IST, Centro de Fusão Nuclear, Lisbon, Portugal \\ ${ }^{4}$ EFDA-CSU, Max-Planck-Institut für Plasmaphysik, Garching, D-85748, Germany \\ E-mail: Christian.Perez.von.Thun@jet.uk
}

\begin{abstract}
The properties of ASDEX Upgrade's type-II ELM regime, which combines good confinement with benign temporal variation of the power exhaust, make it an attractive candidate for ITER baseline scenario operation. As yet it is not understood what the physics origin of this regime is. In previous type-II ELM studies [Stober J et al 2001 Nucl. Fusion 41 1123, Stober J et al 2005 Nucl. Fusion 45 1213] a whole range of fluctuations have been reported. In this article the properties of these fluctuations are inspected in more detail, and their relevance for the regime investigated. For high frequency $(\sim 150-220 \mathrm{kHz})$ magnetic fluctuation activity, from its absence in certain type-II ELM scenarios and behaviour during regime transitions it is concluded that it is not an essential ingredient of type-II ELM regimes on ASDEX Upgrade. A stronger contender to explain the type-II ELM regime appears to be given by small repetitive electromagnetic bursts, and low frequency $(\sim 5-25 \mathrm{kHz})$ magnetic precursor activity associated with these bursts. It is demonstrated that these bursts give rise to small but frequent heat loads of the right order to influence ELM cycles. These are best detected in the vicinity of the inner strike point in the lower divertor through infra-red thermography and Langmuir probes. Their properties further suggest that the bursts are neither small type-I ELMs nor type-III ELMs. However, it remains to be shown that the losses associated with these bursts are high enough to replace the type-I ELM losses. Other enhanced density fluctuation activity at low frequency $(10-30 \mathrm{kHz})$ is detected by reflectometry near the plasma boundary. The information available about this mode is more limited, but so far a causal implication of this mode in the typeII ELM regime cannot be excluded. Overall it can be concluded that, of the three potential dissipation mechanisms, the low frequency electromagnetic burst behaviour seems to be the most promising candidate to account for type II ELMs and their energy losses.
\end{abstract}


PACS numbers: 52.55.Fa, 52.55.Tn, 52.35.Py, 52.55.Rk

Submitted to: Plasma Phys. Control. Fusion 


\section{Introduction}

Small ELM regimes are characterised by small variations in time of the heat fluxes on plasma facing components, thus preventing large peak heat loads as observed during the common type-I ELMs. Such ELM regimes are especially interesting, if they simultaneously show good energy confinement close to the $\operatorname{IPB}(\mathrm{y}, 2)$-scaling. One such regime is the so-called type-II ELM regime in ASDEX Upgrade [1]. This regime is not well understood yet. A whole range of fluctuations has been observed (also varying with the particular type of scenario run), whose relevance is not known. It is not even clear whether the "type-II ELMs" themselves are inherently bursting or continuous phenomena (cf results in [1] and [2]). To understand its physics and the extrapolability to ITER, it is important to identify which modes are necessitated to sustain the regime (e.g. giving rise to enhanced transport across the separatrix).

This paper presents a survey of the MHD activity encountered in the various type-II ELM scenarios documented to date on ASDEX Upgrade. The properties of modes are examined, and their causal relationship with the actual type-II ELM is scrutinised. The paper is structured as follows: Section 2 provides a brief review of the regime. First a list of practical criteria used for regime classification is given. The operational requirements to achieve the regime are outlined, followed by an overview of the various fluctuation types detected. Subsequently, the properties of each of these fluctuations are looked into in more detail. Sections 3 and 4 are dedicated to broadband magnetic activity seen at high $(\sim 150-220 \mathrm{kHz})$ and low $(\sim 5-25 \mathrm{kHz})$ frequency, respectively, while section 5 is devoted to a type of density fluctuations detected with reflectometry. The final conclusions are summarised in section 6 .

\section{Review of regime properties}

\subsection{Criteria for regime classification}

At least two criteria need to be fulfilled in order to classify a regime as a "pure" typeII ELM regime. The first criterion is the absence of large transient heat fluxes to the divertor. This excludes the presence of type-I ELMs. Here, the heat fluxes are diagnosed with a set of two infrared (IR) line-cameras with high temporal (130 or $260 \mu \mathrm{s})$ and spatial (typ. 1.5-6mm) resolution that monitor the temperature in the lower divertor. The second criterion is to have good enough confinement to exclude the possibility of type-III ELMs (which also exhibit small heat fluxes). In practice, this means not more than $\sim 10 \%$ reduction in confinement with respect to the type-I ELM phase. To establish a border between regimes it proves useful to trigger a transition to type-III ELMs within the same discharge by slowly ramping up the gas fuelling. The transition from type-II to type-III ELMs yields a sharp drop in confinement and an increase in divertor recycling (figure $1, t=4.75 \mathrm{~s}$ ). In contrast to this, in a transition from type-I to type-II ELMs the confinement reduction is gradual, with no sudden drop (figure 1, $t=2.5-3.5 \mathrm{~s})$. Mixed regimes of type-I and type-II ELMs have also been observed. 


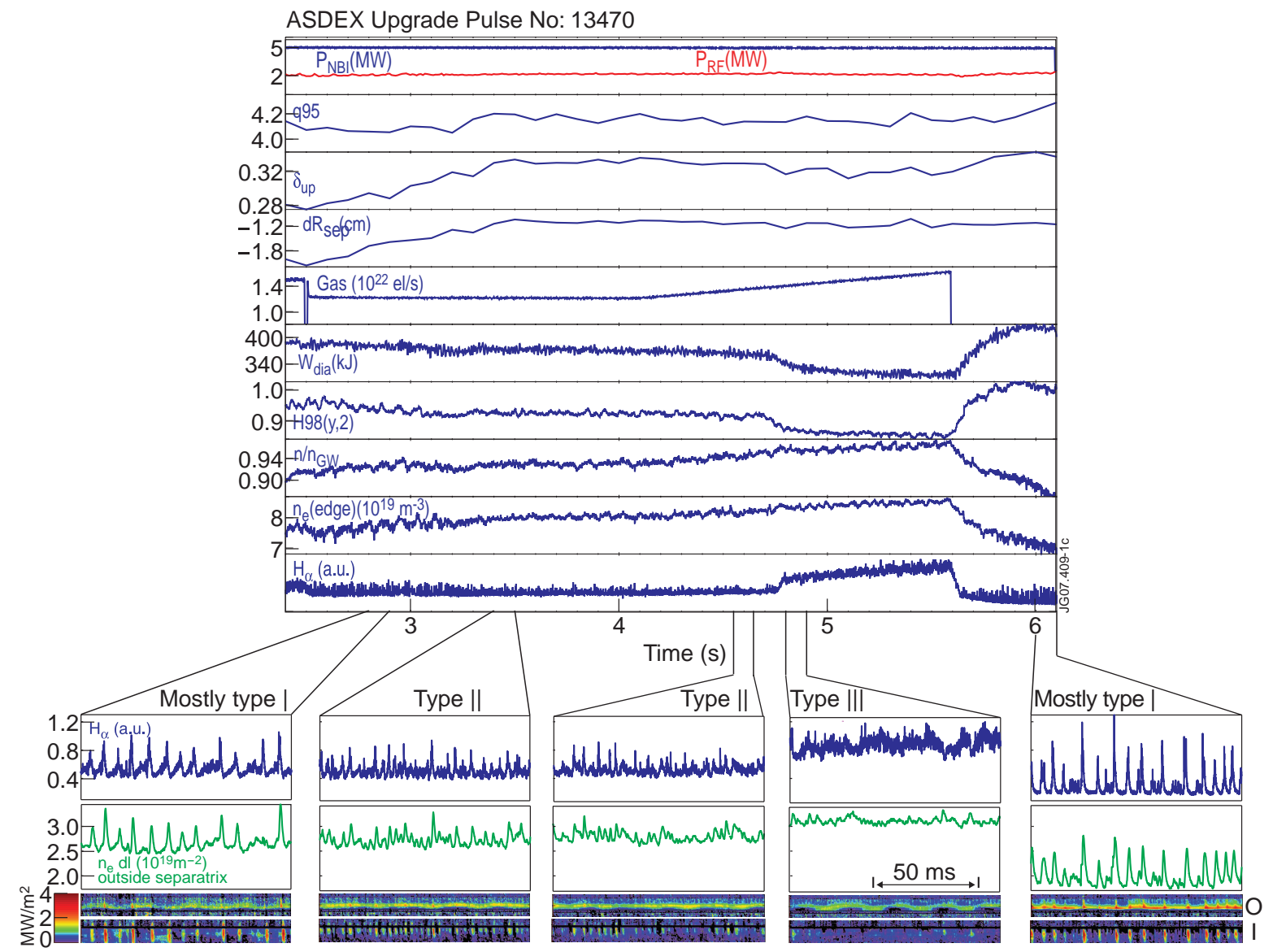

Figure 1. ASDEX Upgrade shot 13470 exhibiting a sequence of ELM regime transitions. (Top): Traces for neutral beam and radiated powers; $q_{95}$; upper triangularity (the lower triangularity, not shown here, remains constant); the radial distance between primary and secondary separatrices at the horizontal midplane, $\mathrm{d} R_{\text {sep }}$ (used to quantify the closeness to double null); deuterium gas fuelling; plasma stored energy; confinement enhancement factor; Greenwald fraction; line-averaged edge density (from interferometry); $\mathrm{H}_{\alpha}$ emission in the lower outer divertor. The transition from type-I to type-II ELMs (2.5-3.5s) is induced by increasing the closeness to DN and/or the upper triangularity. At 4.75s transition to type-III ELMs (induced by increased gas fuelling), which manifests itself through a sudden drop in confinement. Reappearance of type-I ELMs after fuelling switch off (5.6s). (Bottom): $\mathrm{H}_{\alpha}$ emission, line-integrated edge density from an interferometer chord in the scrape-off layer (lineof-sight goes diagonally from below the outer midplane towards the upper dump plate) and fast thermography data for selected time intervals for the outer $(\mathrm{O})$ and inner (I) strike point regions in the lower divertor (where the height of each bar corresponds to a poloidal length of $20 \mathrm{~cm}$ along the target surface). Notice that in general it is not easy to discriminate between type-II and type-III ELMs through the particular shape of $\mathrm{H}_{\alpha}$ fluctuations. On the thermography data the presence of a semicontinuous band of increased heat deposition onto the outer divertor originates from inter-ELM transport and is commonly observed (not specific of type-II ELM regimes). 
There, the presence of type-II ELMs is inferred from the anomalous reduction in type-I ELM repetition frequency.

It would be desirable to have a characteristic MHD signature as a third criterium for the regime. Indeed, in type-II ELM plasmas a number of MHD observations have been made [1,2] identifying particular signatures present with type-II ELMs. On the other hand, cases of type-II ELM discharges have been reported where this MHD activity was missing [2]. The clarification of this issue has motivated this work.

\subsection{Operational requirements}

As has been described in [1], in ASDEX Upgrade type-II ELM regimes have been observed in lower single null plasmas which are run close to double null (DN) and have medium to high average triangularity $(\delta \sim 0.3-0.4)$. Both quantities could not be varied independently (cf figure 1, 2.5-3.5s), but results indicate that the actual key quantity is the closeness to DN. This was concluded from experiments in which the closeness to DN was reduced by a factor of five, while the associated changes in triangularity were comparatively small (about $2 \%$ ). Another requirement is that $q_{95}$ needs to be above a threshold value $(\gtrsim 3.6-4)$. At lower $q_{95}$ only a mixed regime with type-I and type-II ELMs has been encountered. High plasma densities, close to the Greenwald fraction $\left(n_{e} / n_{G W} \geq 0.85\right)$, are beneficial. Increasing the external heating in type-II regime is detrimental and eventually leads to a reappearance of type-I ELMs. In that case, pure type-II ELMs can be recovered by also increasing the gas fuelling.

Type-II ELMs seem not to be sensitive to the type of heating. They have been produced with neutral beam heating, and also with dominant ICRH.

The mass dependence is not well explored. Most experiments were done in deuterium plasmas, although transient type-II ELM phases have also been observed in hydrogen plasmas.

Type-II ELM regimes have also been reported on DIII-D [3,4] and JET [2,5-7]. On DIII-D the suppression of type-I ELMs was achieved in single null (with no mention of DN proximity) by a simultaneous increase of the plasma elongation $(\kappa \gtrsim 1.9)$ and triangularity $(\delta \gtrsim 0.5)$, at high edge safety factor $\left(q_{95} \sim 7\right)$. Experiments on JET have been run in single null and in near double null configurations. With single null $\underline{\left(\delta=0.45-0.50, \kappa=1.75, q_{95}=3\right) \text { a reduction in type-I ELM frequency was observed for }}$ $\underline{n_{e} / n_{G W} \geq 0.7, \text { but a complete type-I ELM suppression has never been achieved (also }}$ not through variation of the edge safety factor, $\left.\left(q_{95}=3-5\right)\right)$. On the contrary, stationary type-II ELM-like regimes free of type-I ELMs have been found more recently with near double null $\left(q_{95}=4.1, n_{e} / n_{G W}=0.7-0.8\right)$. Moreover, a scan in the proximity to double null has shown that double null closeness is indeed important for accessing the regime at JET [7]. 

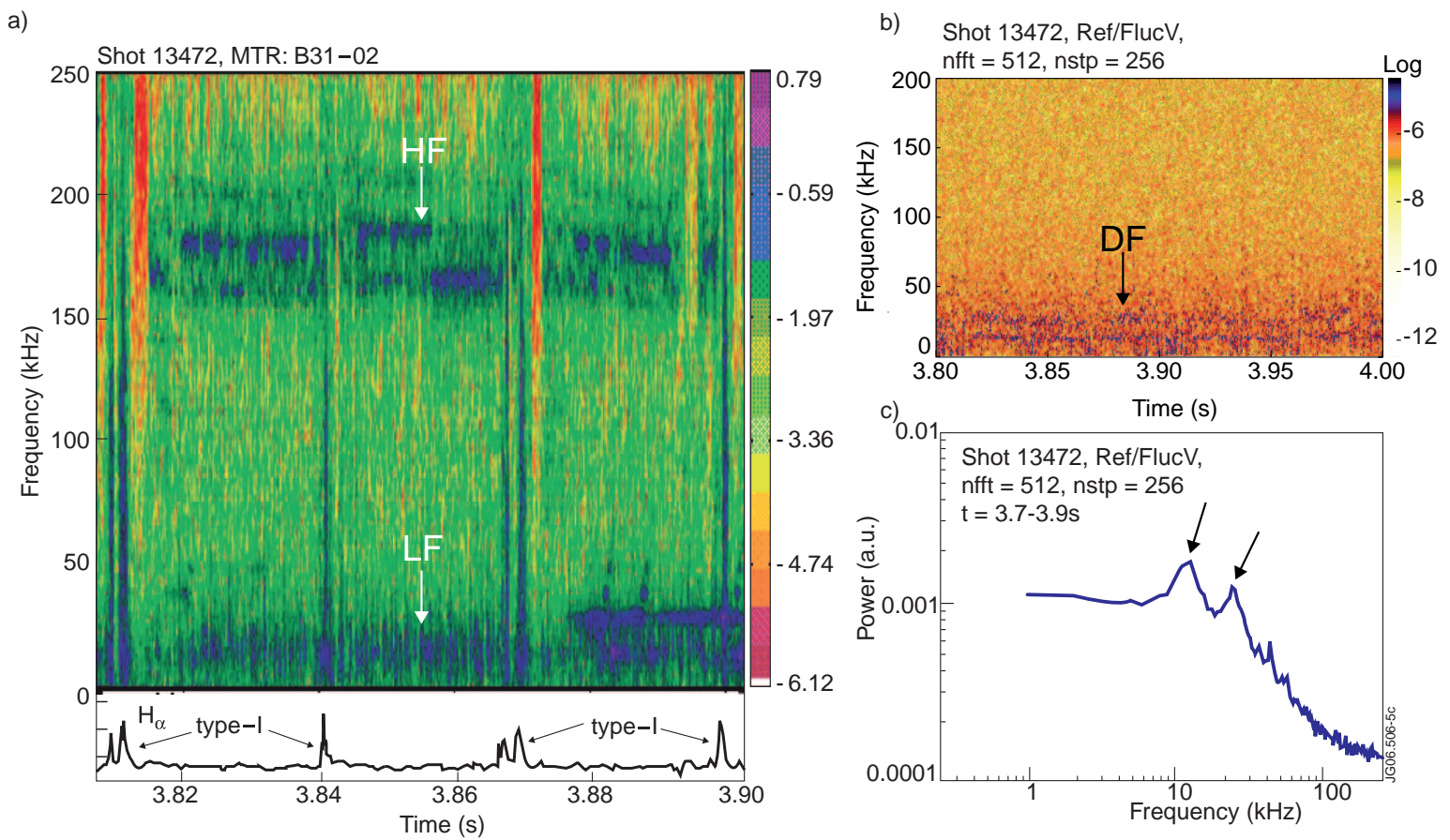

Figure 2. Fluctuation data with type-II ELMs (here mixed with type-I ELMs): (a) spectrogram of magnetic signal, in which the low frequency (LF) and high frequency (HF) activity are marked, (b) spectrogram of reflectometer signal probing near the plasma boundary, where the arrow points the region with enhanced density fluctuations (DF), (c) corresponding frequency power spectrum of edge density fluctuations [1,2].

\subsection{Fluctuation measurements}

Figure 2 brings together previous observations made in connection with type-II ELMs $[1,2]$. Figure 2a shows a magnetic spectrogram (time-windowed Fourier decomposition) from a coil picking up fluctuations on the low field side (a more detailed description of the magnetic fluctuation diagnostic is given in the appendix). This case corresponds to a mixed regime of type-I and type-II ELMs. The type-I ELMs occur at 3.805s, $3.84 \mathrm{~s}, 3.87 \mathrm{~s}, 3.90 \mathrm{~s}$. In the periods between type-I ELMs, increased (rather broadband) fluctuation levels are observable at low frequencies (below $\sim 30 \mathrm{kHz}$ ) and also at higher frequencies $(150-200 \mathrm{kHz})$. Figure $2 \mathrm{~b}$ shows a spectrogram of density fluctuations and figure $2 \mathrm{c}$ the corresponding frequency power spectrum. These are obtained from an O-mode reflectometer (single-ended homodyne detection) probing the plasma from the low field side at fixed frequency [8]. Two such channels are available, one probing in the Q-band (30-50GHz) and one in the $\mathrm{V}$-band $(50-75 \mathrm{GHz})$ frequency range. The cut-off density layer of the channel shown is located close to the separatrix, possibly even in the scrape off layer. Increased density fluctuations are recognisable at low frequencies $(\sim 15 \mathrm{kHz})$. Sometimes also a second peak is resolved at double frequency, presumably the second harmonic of that mode.

Thus we see three fluctuation types that may be linked to type-II ELM behaviour: high frequency magnetic fluctuation activity, low frequency magnetic fluctuation activity and 
low frequency density fluctuation activity. The fact that the fluctuations just outlined are systematically detected in certain type-II ELM scenarios (classified as such following the two criteria outlined above) suggests the possibility of a causal relationship between these fluctuations and the type-II ELM regime. However, it must be stressed that no direct evidence for their involvement has been given so far. The next sections will be dedicated to a more detailed analysis of the fluctuations.

\section{High frequency magnetic fluctuations}

\subsection{Importance for type-II ELM regime}

The enhanced high frequency activity observed in figure $2 \mathrm{a}$ is regularly encountered in type-II regimes at moderate beta values $\left(\beta_{\mathrm{N}} \sim 2, \beta_{\mathrm{pol}} \sim 1\right)$, but it is no longer detected if the heating is increased further. Standard deuterium type-II ELM discharges are run with $5 \mathrm{MW}$ auxiliary heating (two NBI sources). In standard baseline scenario discharges utilising 7.5 MW (i.e. one additional source) the high frequency turbulence already appears to be gone. Likewise, hybrid scenario discharges with type-II ELMs [9] (which are run with $10 \mathrm{MW}$, or more) do not show the high frequency activity either. Reference [2] has pointed at the possibility that the activity is still present, but not seen because the overall background fluctuation level is higher in these discharges. This issue has been further investigated. Hybrid discharges are routinely run down such that after the high power phase there is a transient phase with lower heating. While not being detected in the high power plateau, it has been found that in this transient phase the enhanced high frequency activity is clearly detected. In figure 3 (right plot) two magnetic power spectra are plotted, one corresponding to the high power phase, and the other one for the low power phase with the enhanced high frequency activity. It becomes clear that although the background fluctuation level is higher with increased heating, the high frequency turbulence is well above those levels. This has been confirmed for total input powers up to at least $16 \mathrm{MW}$. Hence, it is concluded that the high frequency activity is not covered up by the noise but truly absent in type-II ELM regimes at high beta.

If the type-II ELM regime is induced by the high frequency fluctuations, then one would expect to measure a gradual amplitude enhancement during transitions from type-I to type-II ELMs. Figure 4 shows magnetic power spectra for two time windows of the same discharge, one with pure type-II ELMs and one with a mixed regime of type-I and type-II ELMs. In this discharge the transition from type-II to type-I ELMs is induced through a slow ramp down in gas fuelling at fixed $q_{95}$ (hence the poloidal mode number of the fluctuations, which are important for the radial decay of magnetic perturbations, remain unchanged). There is no obvious amplitude enhancement in the pure type-II regime. In fact, the fluctuation amplitude has even decreased, by approximately $30 \%$ in this case.

Furthermore, the enhanced high frequency activity has not been observed in hydrogen 

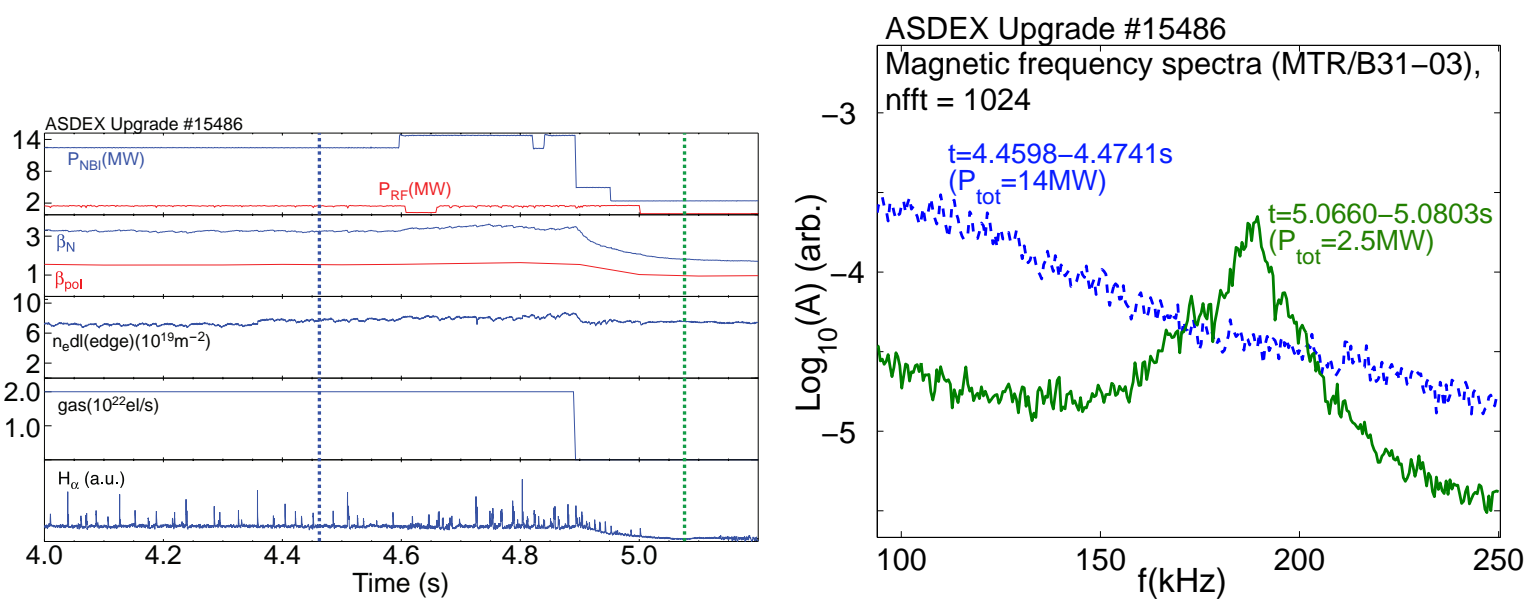

Figure 3. (Right) Amplitude of the high frequency activity in the final low power phase with respect to the background noise level in the high power phase (in mixed type-I/type-II ELM regime) of a hybrid scenario discharge. A selection of traces for that discharge with the relevant times marked by vertical lines is shown in the left plot.
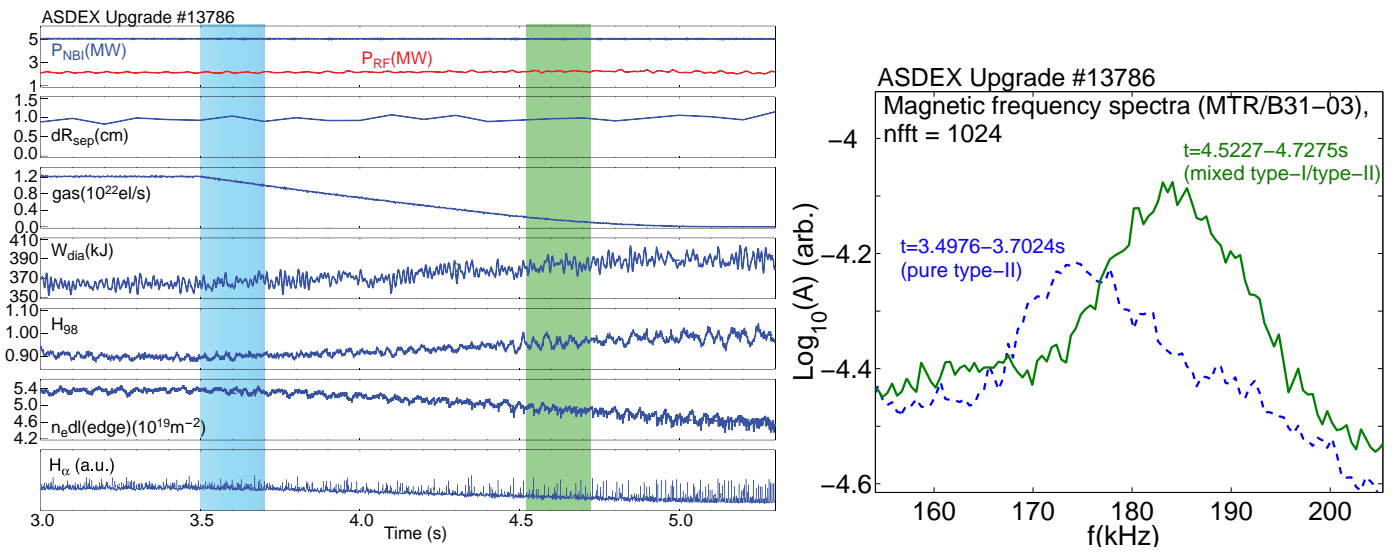

Figure 4. Amplitude of high frequency magnetic fluctuations for a transition from pure to mixed type-II ELM regime induced through density variation (slow ramp down in gas fuelling). The blue and green coloured bars in the left plot mark the respective time intervals used for the frequency spectra. No amplitude enhancement is observed when going from mixed (green curve) to pure (blue curve) type-II ELM regime.

discharges with type-II ELMs, also at moderate beta.

The above evidence is not supportive of the high frequency magnetic fluctuation activity being an essential ingredient of ASDEX Upgrade's type-II ELM regime. It is also worth mentioning that Stober et al [2] has speculated whether the small ELM regime observed in the hybrid scenario and so far classified as type-II could be in reality a grassy ELM regime [10] (which on ASDEX Upgrade have been observed at similar high beta values and similar plasma shape, but significantly higher $q_{95} \sim 6.5$ [11]). This offers the possibility that the small ELM regime at high beta is different to the type-II ELM regime at moderate beta. If the mechanisms are different, then one could speculate further and 

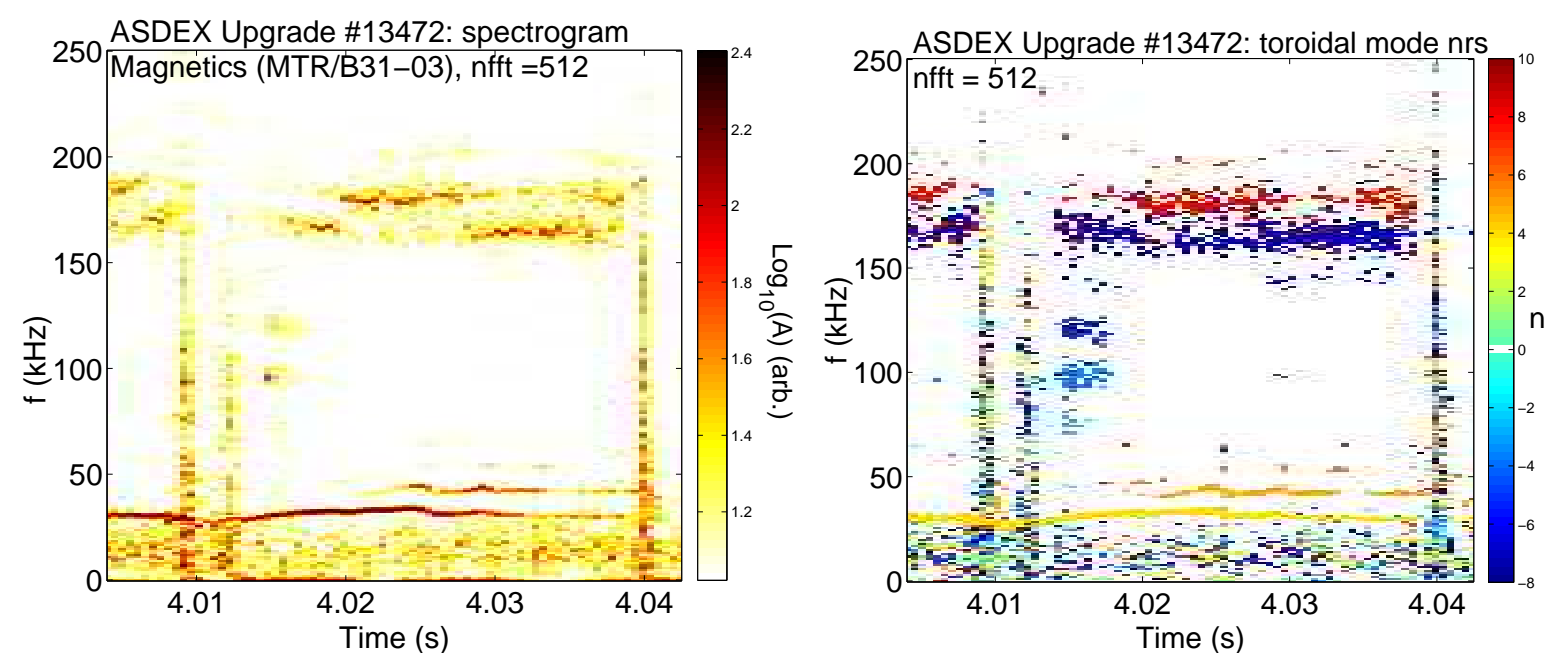

Figure 5. Magnetic spectrogram (left), and the corresponding toroidal mode numbers, $n$ (right).

state that the high frequency mode is important for the moderate beta regime but not for the high beta regime. However, even this hypothesis is not supported by the present data, since figure 4 indicates that the activity is not necessitated also at moderate beta.

\subsection{Mode identity}

Figure $5 \mathrm{~b}$ gives the toroidal mode numbers $n$ of fluctuations versus time and frequency (for the same discharge as in figure 2). The mode number spectra are obtained by analysing the relative phase shift of fluctuations detected by a set of toroidally distributed coils (see appendix). Modes with negative $n$ rotate in the opposite direction to modes with positive $n$ (the convention used here is that modes with negative $n$ rotate in the direction of the electron diamagnetic drift). It can be seen that the high frequency activity is here essentially composed of two bands. The lower of the two has $n=-7$ and hence rotates in the electron drift direction. The higher band (red coloured) suffers from aliasing (the natural phase difference between the two closest coils approaches $\pi$ ) and has in reality $n=-8$ (in this shot one of the magnetic pick-up coils was not functioning; when this coil works the aliasing is no longer observed). This agrees with the range of mode numbers reported earlier for the high frequency turbulence [2]. Most interestingly, a shortlived series of bands is discernable at 4.015s (early ELM period) with $n=-3$, $-4,-5$ and -6 , resembling the standard appearance of washboard (WB) modes on JET $[12,13]$. At this time the bands with -7 and -8 represent the natural continuation towards higher mode numbers and frequency. For some reason yet to be determined, the bands with lower mode numbers abruptly disappear, and only the bands with $n=-7$ and -8 survive. In addition the amplitude of the two remaining bands enhances (see spectrogram). This pattern could be also observed in other type-II ELM pulses, and suggests that the high frequency fluctuations originate from the same type of instability as the WB modes with high $n$ and enhanced amplitude that have been observed in 
JET's "mixed type-I/type-II ELM regime" [5].

In this context it is worth mentioning that only very recently it has been identified that washboard-like modes are present in ASDEX Upgrade. "Standard" (so, not conspicuously enhanced) washboard modes are found to be a fairly ubiquitous property of ASDEX Upgrade H-mode regimes. This aligns with earlier JET findings, again indicating that the modes on JET and ASDEX Upgrade share the same physics mechanism. The reader is referred to [14-16] for more details.

\section{Low frequency magnetic fluctuations}

\subsection{Phenomenology}

The low frequency magnetic activity is composed of a quick succession of small repetitive magnetic bursts, which in spectrograms manifest themselves as a series of (more or less extended) vertical lines, and precursor activity associated with these bursts. Figure 6 shows data for a sequence of bursts. Because of the high repetition rate of the bursts combined with the finite lifetime of the precursor, on the spectrogram the precursor activity is seen here to form a quasi-continuous band. Also included in figure 6 is a zoomed view for a single burst. Prior to the burst the growth rate of the precursor is clearly accelerating, indicating that the precursors indeed trigger the bursts.

The precursor can be detected on the low or on the high field side, or on both. At high beta (hybrid scenario discharges with type-II ELMs) it is found on the high field side only (figure 7). Due to the different coil types used (see appendix), it has not been possible to determine whether this is due to the higher background fluctuation level on the low field side, or whether under these conditions the precursor amplitude becomes antiballooning.

\subsection{Induced losses}

The thermography data in figure 6 demonstrates that the small bursts give rise to small but frequent heat loads onto the lower divertor. These are also visible in the thermography data in figure 1 (close to the inner strike point).

Please note that in the thermography data in figure 1 (and also in figure 6 ) the presence of a semi-continuous band of increased heat deposition near the outer strike point is due to inter-ELM losses (with which we mean here the continuous losses occurring between bursts of any kind). It is known that on ASDEX Upgrade inter-ELM losses flow predominantly to the outer divertor (provided the $\nabla B$-drift is pointing towards the X-point) [18]. Although this power deposition pattern is common and not specific of type-II ELM regimes, the magnitude of the continuous background of this band may very well change in type-II ELM regimes, signalling changes in the level of continuous power exhaust. This point will be addressed further below.

In practice the heat loads associated with the small bursts are much easier to detect on the inner divertor than on the outer divertor, for two reasons. Firstly, surface effects [19] 


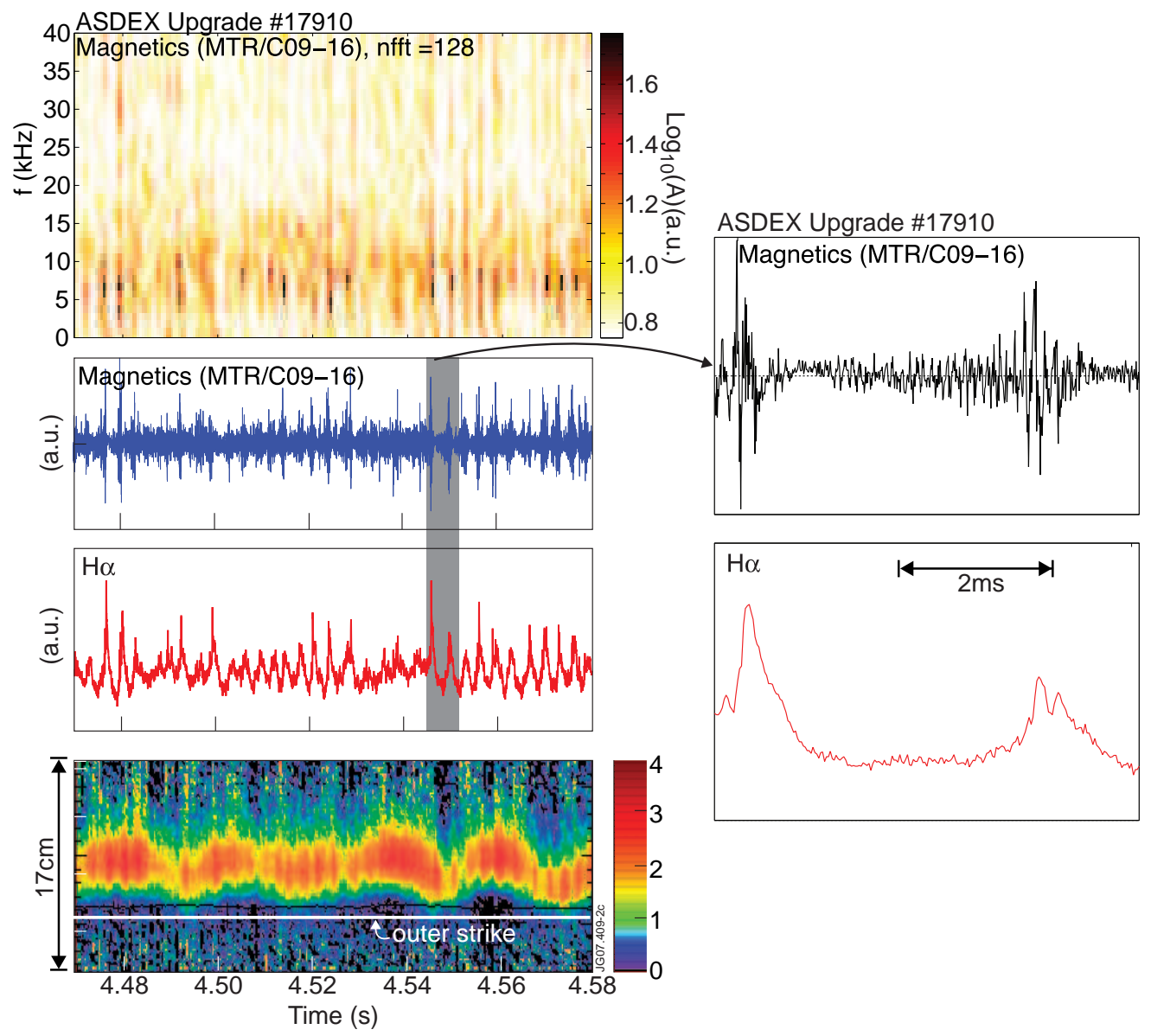

Figure 6. Study of small bursting activity in pure type-II ELM regime (shot 17910). Shown are: Magnetics spectrogram, the corresponding magnetic signal, divertor $\mathrm{H}_{\alpha}$ emission and thermography data for the outer divertor (where the strike point position is from a CLISTE equilibrium reconstruction [17]). In the thermography data, the burst-associated losses are recognisable as slight modulations in the power density of the continuous inter-ELM deposition band. On the right hand side, a closer view of the magnetic trace showing the precursor fluctuations prior to a burst, plus $\mathrm{H}_{\alpha}$ trace.

that are more pronounced for the CFC material of the inner divertor compared to fine grain graphite at the outer divertor. These surface effects can be expressed as thermal resistance which is typically $5-8 \mathrm{~K} \mathrm{MW}^{-1} \mathrm{~m}^{2}$ for fine grain graphite and 10$30 \mathrm{~K} \mathrm{MW}^{-1} \mathrm{~m}^{2}$ for CFC. Secondly, a closer analysis of the total energy deposited by the bursts on the inner and the outer divertor (which is not affected by the surface effects [20]) shows that the losses flow indeed predominantly to the inner divertor. Spurious reflections of light coming from a different location inside the vessel can be excluded as a source for the detection on the inner divertor, since the incoming fluxes have also been detected there by Langmuir probes (figure 8).

Also in figure 8 it can be seen that, compared to the outer upper and outer lower divertor, the $\mathrm{H}_{\alpha}$ emission on the inner lower divertor is delayed. This indicates that the bursts originate on the low field side, and then propagate towards the high field side. 

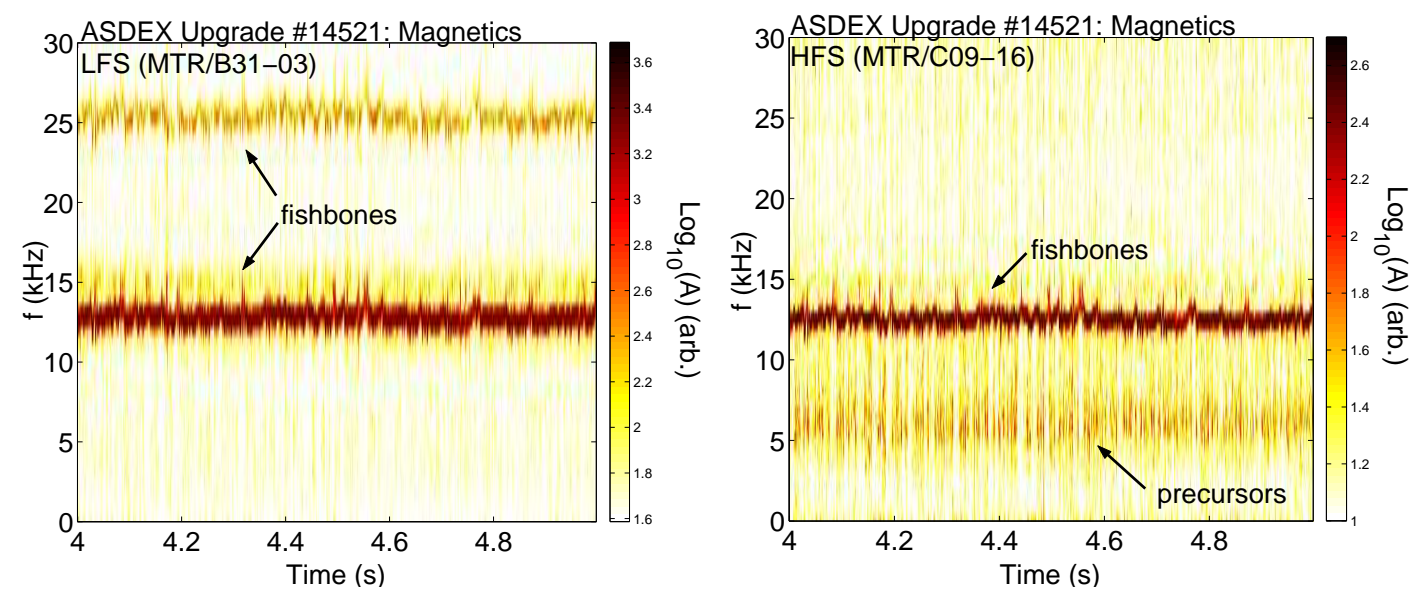

Figure 7. Low frequency magnetic fluctuations in hybrid scenario: Magnetic spectrogram from coil on LFS (a), and from coil on HFS (b). The precursor fluctuations associated with the small bursts are only visible on the HFS, around $6 \mathrm{kHz}$.

ASDEX Upgrade Pulse No: 13470
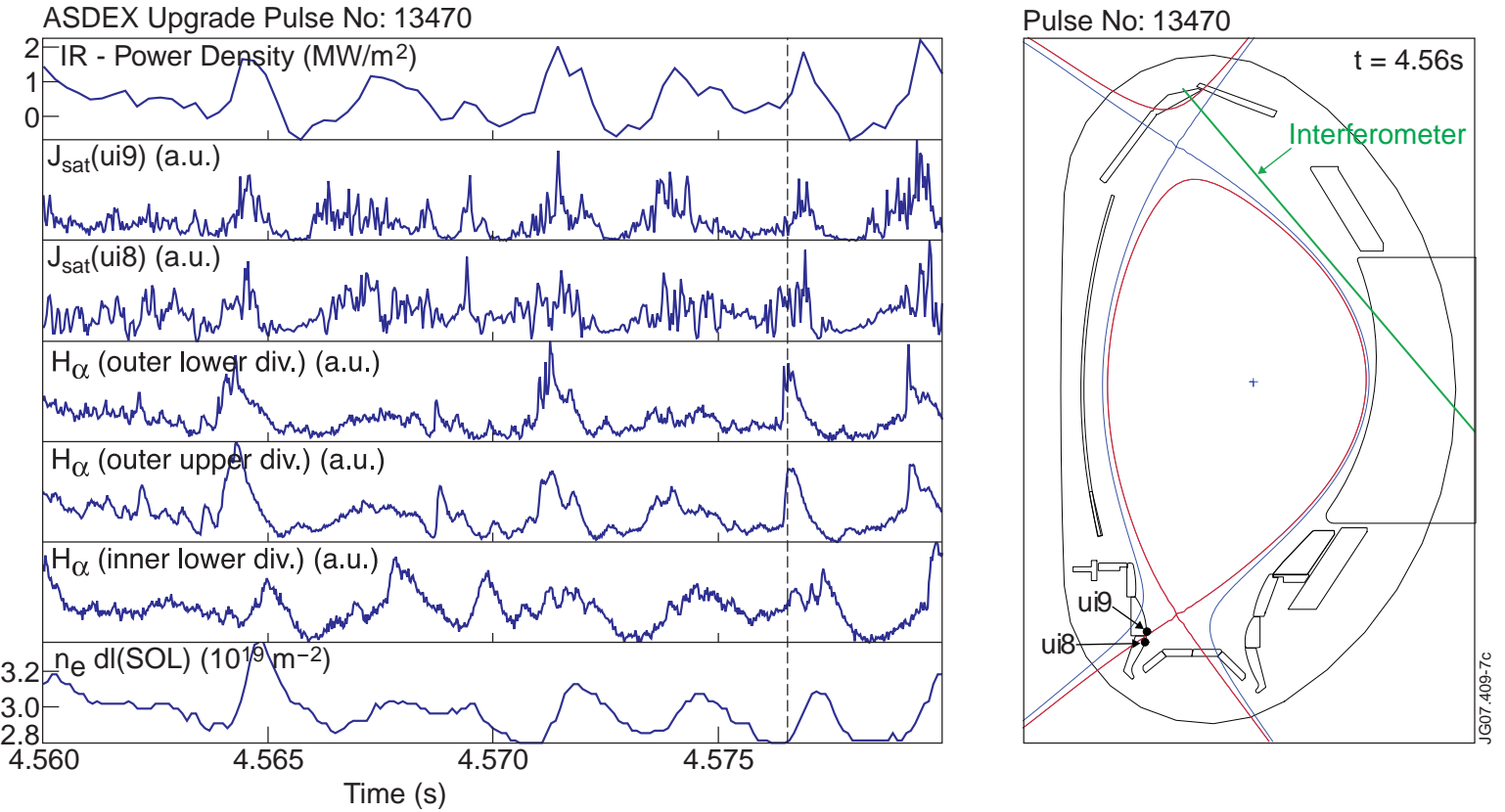

Figure 8. Identification of small bursts in pure type-II ELM regime (shot 13470) on different diagnostics. Shown are: power density deposited approx. $2.5 \mathrm{~cm}$ above the inner strike point, obtained with thermography; ion saturation current from two Langmuir probes in the inner divertor, again close to the inner strike point (their exact position is shown in the plot to the right); $\mathrm{H}_{\alpha}$-emission at three different locations; line-integrated density from a DCN interferometer chord in the scrape-off layer (its line-of-sight is shown in the plot to the right). On the right hand plot, the active separatrix is shown in red, and the secondary separatrix in blue. 


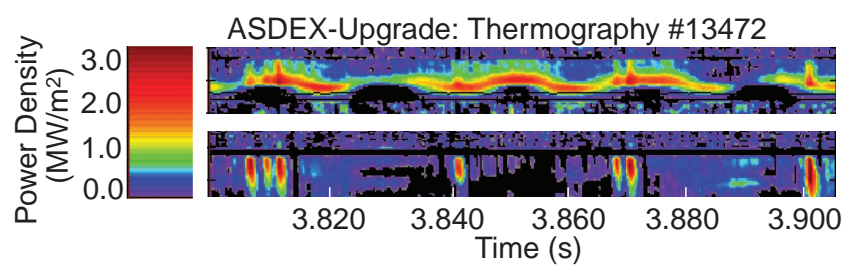

ASDEX-Upgrade: Thermography \#13470
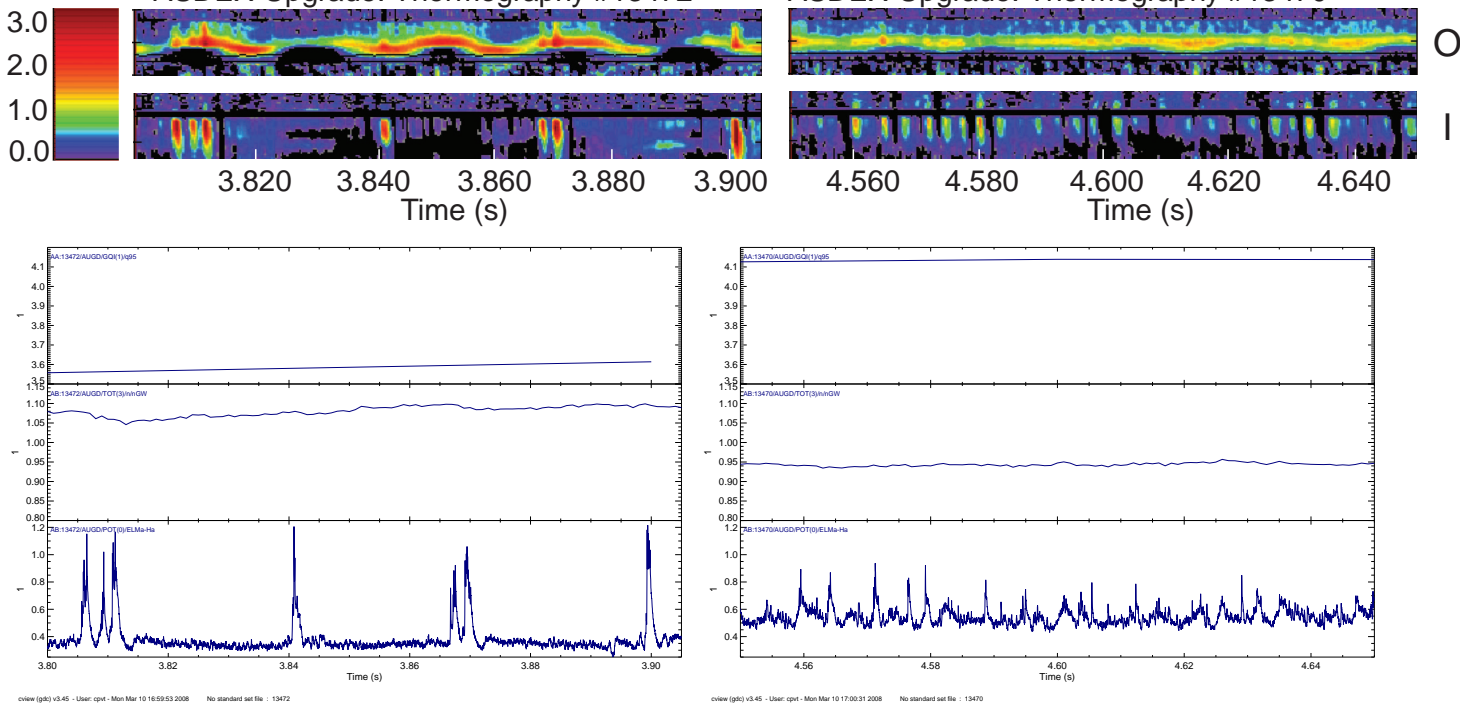

Figure 9. Transition from mixed type-I/type-II to pure type-II ELM regime through $q_{95}$ variation: Heat flux to the outer $(\mathrm{O})$ and inner $(\mathrm{I})$ strike point regions, from infrared thermography. The height of each bar corresponds to a poloidal length of $20 \mathrm{~cm}$ along the target surface. The transient heat loads visible for shot 13472 are due to type-I ELMs. The continuous black line on the inner divertor is due to a small $(2.1 \mathrm{~cm}$ wide) gap in diagnostic coverage. The plasma parameters of the two discharges are as follows: (13472) $B=1.6 \mathrm{~T}, \quad I=800 \mathrm{kA}, \quad q_{95}=3.6, \quad P_{\mathrm{nbi}}=5 \mathrm{MW}, \quad n_{e}($ edge $)=9.0 \times 10^{19} \mathrm{~m}^{-3}$,

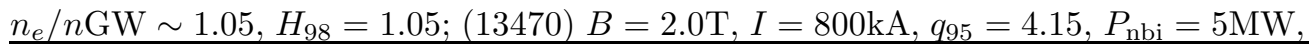
$\underline{n_{e}(\text { edge })=8.0 \times 10^{19} \mathrm{~m}^{-3}, n_{e} / n \mathrm{GW} \sim 0.95, H_{98}=0.96 .}$

Another independent measurement of the bursts is provided by interferometry. Figure 8 (and also figure 1) shows data from one of the chords of the DCN interferometer system that for this particular shape runs entirely in the scrape off layer (on the low field side) and the upper divertor region. It detects the temporal density rise caused by each burst in the region outside the separatrix. Compared to the thermography and the Langmuir probe data, the bursts on the interferometer occur slightly later (about 1ms delay). The reason for this delay is not understood, since the expelled electrons should be propagating very fast. Nevertheless, the delay is not specific of these bursts, since a similar delay is also observed for type-I ELMs.

\subsection{Importance of bursts for type-II ELM regime}

The small magnetic bursts are the only activity that has been robustly observed in all type-II ELM scenarios run on ASDEX Upgrade. To get further insight into the causality, the burst behaviour in regime transitions has been examined. Since to retain stationary conditions the type-I ELM induced losses need to be replaced by other losses, in a transition from mixed to pure type-II ELM regime the key activity should be seen to enhance. Figure 9 shows thermography data for a pair of discharges in which $q_{95}$ was scanned (toroidal field variation at fixed plasma current). Despite having slightly 

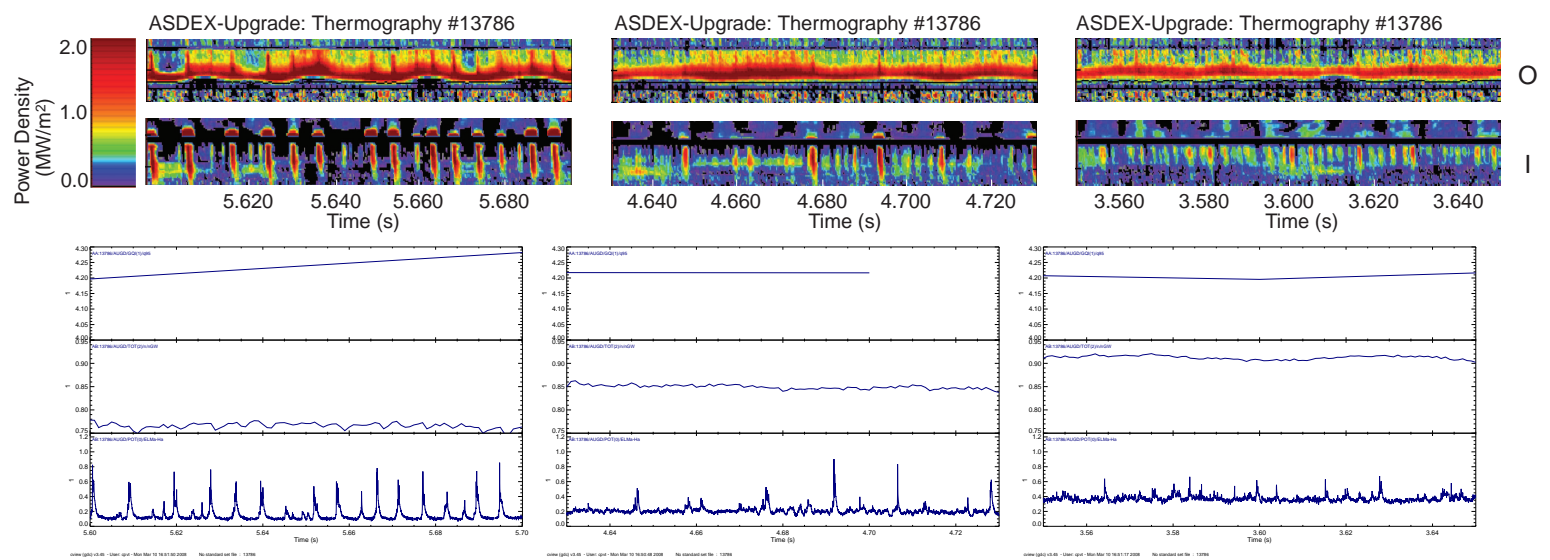

Figure 10. Heat flux to the outer $(\mathrm{O})$ and inner $(\mathrm{I})$ strike point regions for a gradual transition from type-I to type-II ELM regime (from left to right) induced through a slow ramp in gas fuelling (corresponding Greenwald fractions are shown below: 0.77, $0.85,0.91)$. On the thermography data the height of each bar corresponds to a poloidal length of $20 \mathrm{~cm}$ along the target surface.

higher density (which favours accessing the type-II), discharge $13472\left(q_{95}=3.6\right)$ reaches a mixed regime of type-I and type-II ELMs only, while $13470\left(q_{95}=4.15\right)$ has pure type-II ELMs. In 13472 the burst associated peak power density detected on the inner divertor is barely visible and remains below $0.6 \mathrm{MW} / \mathrm{m}^{2}$, while in 13470 reaches values of $1-2 \mathrm{MW} / \mathrm{m}^{2}$.

Figure 10 shows a similar survey where the transition has been induced through a slow ramp in gas fuelling. Again, with the gradual disappearance of the larger type-I ELM losses, the heat deposition associated with the small bursts becomes increasingly visible on the inner divertor.

These findings are in line with a causal implication of the small bursts in the type-II ELM regime. However, it could not be determined whether the burst losses are indeed high enough to completely replace the type-I ELM losses. At these high repetition frequencies the diamagnetic loop measurements no longer resolve the drop in stored energy. An alternative way is to make use of scrape-off layer diagnostics to quantify the losses. Since a full energy assessment would require thermography measurements of the upper divertor as well as ELM-resolved radiated energies, which are not available, only a partial assessment can be given. Table 1 gives the exhausted powers during the type-I and the type-II ELM phase for discharge 13786 (cf. figure 4), inferred from lower divertor thermography (where the power deposited has been corrected for the radiation contribution [21]) and the reconstructed 2-D radiation profiles from bolometry (ELM-averaged). The first two rows give the powers dissipated in the lower outer and lower inner divertor, respectively. It can be seen that during the type-II ELM phase the total power to the outer divertor is essentially the same as for the type-I ELM phase, despite the disappearance of the type-I ELMs. This is due to an enhancement of the continuous deposition (incl. radiation) during the type-II ELM phase. Since in the type-II ELM phase the contribution of the small bursts for the outer divertor 


\begin{tabular}{|l|c|c|}
\hline AUG shot 13786: & $3.3-3.5 \mathrm{~s}($ type-II) & $5.5-5.7 \mathrm{~s}($ type-I) \\
\hline power to lower outer div. & $1.64 \mathrm{MW}$ & $1.68 \mathrm{MW}(*)$ \\
\hline power to lower inner div. & $0.46 \mathrm{MW}$ & $0.58 \mathrm{MW}$ \\
\hline$P_{\text {inp }}-P_{\text {rad }}(\rho<1)-P_{\text {lower-div }}$ & $1.70 \mathrm{MW}$ & $1.55 \mathrm{MW}$ \\
\hline
\end{tabular}

(*) out of which 0.15-0.20 MW due to type-I ELM deposition

Table 1. Dissipated (deposited + radiated) powers for two time intervals of discharge 13786. The first interval corresponds to a type-II ELM regime, and the second to a type-I ELM regime.

is found to be negligibly small, it is speculated that this transport increase is induced by the high frequency magnetic fluctuation activity (section 3) or the low frequency density fluctuation activity (section 5). On the inner divertor the power dissipated in the type-II ELM phase is found to be only slightly smaller than in the type-I ELM phase. Despite the uncertainties with the radiation contribution between the bursts, this result indicates that the losses induced by the small bursts are of the right order to replace the type-I ELMs, and should have an effect on the ELM cycle. The third row contains the total input power, $P_{\text {inp }}$, minus the power radiated inside the separatrix minus the power dissipated in the entire lower divertor. This quantity is taken as a reference for the power flowing to the upper dump plates, which could not be directly measured. It is slightly higher during the type-II ELM phase than during the type-I ELM phase. If originating from the small bursts, then this difference would compensate the power reduction on the inner divertor. Indeed, $\mathrm{H}_{\alpha}$ measurements show that at least some of the energy originating from the small bursts arrives in the upper divertor. Nevertheless, further improd measurements the to quantify this thermography measurements of the upper divertor are required. A 2-D infrared camera which monitors the upper dump plates has been installed on ASDEX Upgrade more recently, so future type-II ELM experiments should be able to address this issue.

Based on the data given in table 1 , it is now attempted to give a crude estimate of the energy expelled by each of the small bursts for the case of discharge 13786. In the first estimate we assume that the energy expelled by the bursts flows entirely to the inner divertor, and that the energy dissipated there (table 1) originates entirely from the small bursts. Since we neglect the radiation contribution between bursts, this should be considered a worst case scenario. In the second estimate we effectively double the burst energy by assuming that the bursts deposit their energy equally onto the inner divertor and the upper target. Apart from the dissipated powers, the other quantity needed is the repetition frequency of the bursts. In practice, this is not easy to determine. Compared to the thermography data, which has a more limited time resolution (260 $\mu$ s sampling), the $\mathrm{H}_{\alpha}$ traces (25 $\mu$ s sampling) are able to resolve a higher number of events. This can actually be discerned in figure 8, particularly towards the beginning of the time window. Depending on whether one regards those as subevents of a burst, or whether each $\mathrm{H}_{\alpha}$ event is counted as a burst on its own, one obtains for 13786 (3.3-3.4s) 
burst repetition frequencies of $600 \mathrm{~Hz}$ or $2.1 \mathrm{kHz}$, respectively. Correspondingly, the average energy released per event ranges from $3.7-1.1 \mathrm{~kJ}$ (first estimate) to $7.4-2.2 \mathrm{~kJ}$ (second estimate). The stored energy of the plasma is $400 \mathrm{~kJ}$. Hence, our estimates yield an average relative energy loss per event $\left(\Delta W / W_{t o t}\right)$ which ranges from 0.3 to $1.9 \%$. Overall we can say that the energy loss should lie below $2 \%$, and that, even though it would be desirable for ITER considerations, with the available diagnostics it is difficult to give more precise estimates.

\subsection{Mode identity}

From the point of view of energy deposition, the small bursts could well be interpreted as small type-I ELMs, since for standard $\nabla B$-drift direction (pointing towards the X-point), type-I ELM losses also go preferentially to the inner divertor $[18,22,23]$. However, with co-injection no precursor activity is observed for type-I ELMs on ASDEX Upgrade [24,25], which is different to the observations made for the small bursts. TypeIII ELMs may have precursors [25], but, in contrast to the small bursts, the induced losses go preferentially to the outer divertor [18]. Also the confinement behaviour outlined in section 2.1 is not supportive of type-III ELMs. Hence, the above properties suggest that the bursts are neither small type-I ELMs nor type-III ELMs.

\section{Density fluctuations}

\subsection{Phenomenology}

In reference [1], the enhanced fluctuations on the reflectometry signals were regarded as the density counterpart to the low frequency magnetic fluctuations treated in section 4 . This is because the range of frequencies was comparable for the two cases. A closer inspection reveals that the respective fluctuation frequencies actually do not coincide (figure 11a). This indicates that the activity seen on the reflectometry and the magnetic signals are caused by different instabilities (in which case the density fluctuations would either be electrostatic, or have such high poloidal mode numbers that the magnetic perturbations decay radially away before reaching the magnetic sensor). An alternative explanation could be that, since the reflectometer measurement is highly localised, while the magnetic probe sees a much wider radial range, the results need not be identical in the presence of several unstable modes at the plasma edge. However, in that case one would expect the coherence of the two signals to attain a high value at the same fluctuation frequencies. As shown in figure 11a, this is not the case. The coherence remains below $0.2-0.3$.

\subsection{Importance for type-II ELM regime}

Figure 11b shows two spectra from reflectometry for discharge 13470, for the transition from type-II to type-III ELMs shown in figure $1(t=4.75 \mathrm{~s})$. The green and the purple 

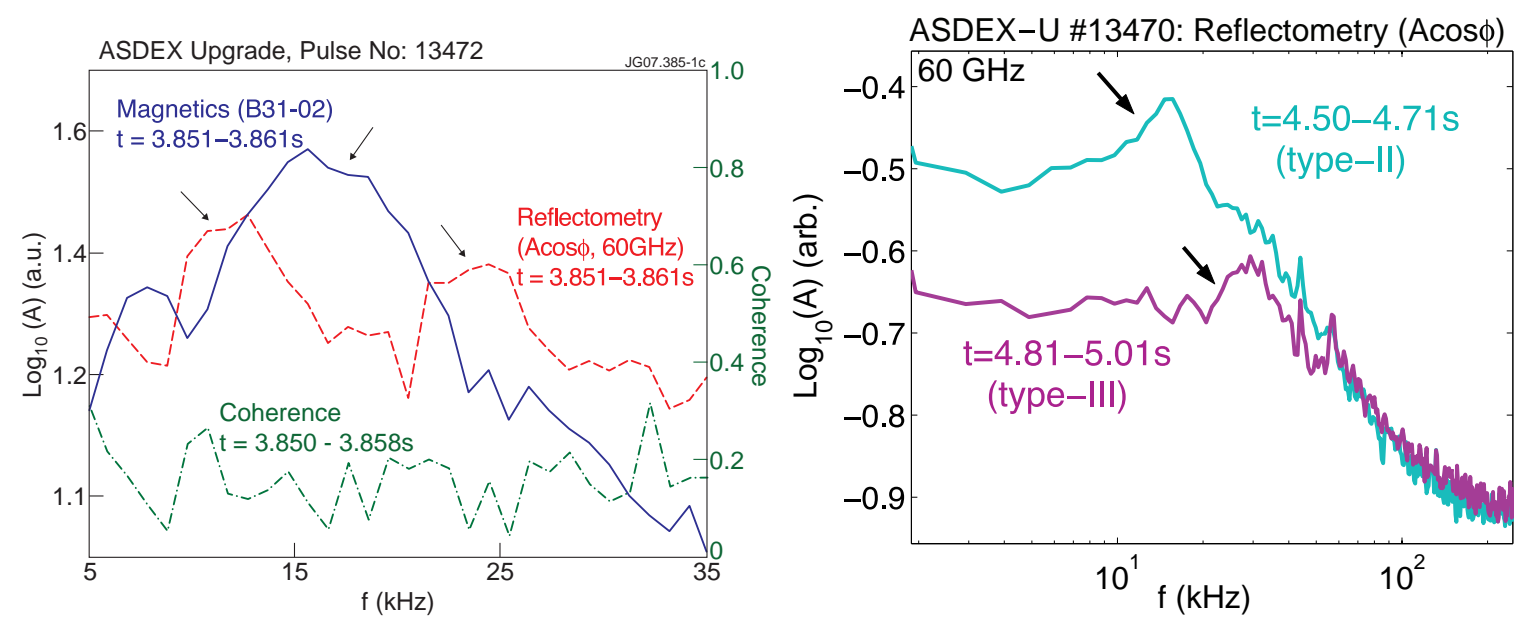

Figure 11. (a) Frequency spectra from magnetics (solid) and reflectometry (dashed) for a time window of discharge 13472 (in mixed type-I/type-II ELM regime, cf figure 2). The respective fluctuation frequencies do not coincide. The dash-dotted curve gives the coherence of the two signals. (b) Reflectometry spectra for discharge 13470, just before and after a transition from type-II to type-III ELMs. The enhanced fluctuation activity is marked by arrows. Both in (a) and (b) the reflectometer is probing in the upper half of the steep gradient zone.

curves correspond to times just before and after the transition, respectively. Edge density profiles have been evaluated with a lithium beam diagnostic, and show little variation for these particular times, hence the two spectra may be directly compared. On the one hand it can be seen that the density fluctuation activity persists after the transition, shifted towards higher frequencies (30 versus $16 \mathrm{kHz}$ ). A separate analysis yields that the fluctuations continue across the whole type-III ELMy phase, $t=4.8-5.6 \mathrm{~s}$. This implies that their existence domain is not restricted to type-II ELM regimes. On the other hand it can be seen that the amplitude of the remaining fluctuations is reduced compared to the phase with type-II ELMs. Hence, while it is not possible to be more conclusive with the present data, a causal implication of the density fluctuation activity in the type-II ELM regime cannot be excluded. A frequency hopping millimeter-wave reflectometer [26] installed in ASDEX Upgrade is now capable of measuring density fluctuations profiles with a fast $(\sim 100 \mathrm{~ms})$ time scale. Recent results have been obtained for the determination of the radial eigenfunction of MHD and fast particle modes [27]. As a future work, the radial structure of the MHD modes present in type II-ELM discharges could be determined in the pedestal region. However, the radial coverage is limited due to the maximum probing frequency of $75 \mathrm{GHz}$. This problem can be overcome by the use of X-mode or by adding a new frequency band.

\subsection{Induced losses and related JET observations}

On ASDEX Upgrade no proof of enhanced losses directly attributable to this mode has been found. It is worth noting that similar density fluctuation activity has been 
found on JET in identity experiments designed to match ASDEX Upgrade discharges with type-II ELMs $[6,7]$. It could be shown that the mode on JET is responsible for increased particle transport across the separatrix, while it is not known in how far the edge temperature is also affected [28]. Time lags for the onset of enhanced $\mathrm{H}_{\alpha}$ emission at different poloidal locations and their interpretation in terms of the finite parallel transit time of ions in the SOL imply that the temperature of ions released by the density fluctuations is nearly an order of magnitude smaller than those expelled by nearby type-I ELMs. Some similarities with the quasi-coherent mode [29] of Alcator C-Mod were found [28].

\section{Summary and Discussion}

The properties of fluctuations encountered in ASDEX Upgrade's type-II ELM regime have been examined. Three types of fluctuations have been observed: high frequency magnetic fluctuation activity, small repetitive magnetic bursts and low frequency density fluctuations.

For the high frequency $(\sim 150-220 \mathrm{kHz})$ magnetic fluctuation activity similarities to JET's enhanced washboard modes have been established. Although JET experience suggests that this type of activity leads to enhanced (conductive) inter-ELM losses and a reduction of type-I ELM frequency [12], from their absence in certain type-II ELM scenarios and their behaviour during regime transitions it appears to be that this activity is actually not an essential ingredient of type-II ELM regimes on ASDEX Upgrade.

Another type of activity is given by small repetitive bursts, and low frequency $(\sim 5$ $25 \mathrm{kHz}$ ) magnetic precursor activity associated with these bursts. The small repetitive bursts are the only activity that has been robustly observed in all type-II ELM scenarios run on ASDEX Upgrade. It has been identified that these bursts give rise to small but frequent heat loads onto the lower divertor, mainly in the vicinity of the inner strike point, which have been detected through infra-red thermography and Langmuir probes. These are of the right order of magnitude to have a significant effect on ELM cycles. The particle losses associated with these bursts lead to a temporal density rise in the region outside the separatrix which has been detected with interferometry. $\mathrm{H}_{\alpha}$ measurements at different poloidal locations suggest that the losses originate on the low field side, and then propagate towards the high field side. The bursts appear neither to be small type-I ELMs nor type-III ELMs. The burst behaviour during regime transitions is found to be consistent with a causal implication of these bursts in the type-II ELM regime. However, it remains to be clarified whether the losses associated with these bursts are sufficiently high to replace type-I ELM losses, or whether additional loss mechanisms are necessary.

The third activity is given by enhanced density fluctuations at low frequency (10-30 $\mathrm{kHz}$ ) detected by reflectometry near the plasma boundary. Evidence has been given supporting the idea that these fluctuations are not simply the density counterpart to the low frequency magnetic fluctuation activity, but originate from a different type of 
instability. The information available about this mode is more limited, but so far a causal implication of this mode with the type-II ELM regime cannot be excluded. A similar type of activity has been observed in JET in identity experiments designed to match ASDEX Upgrade discharges with type-II ELMs [28], where it could be associated with enhanced particle losses.

Assuming that the small repetitive bursts, which from the three candidate modes appear to be the strongest contender, are indeed the type-II ELMs, it can be attempted to compare the experimental observations with current type-II ELM models. Modelling calculations performed by Saarelma et al [30], which use experimentally measured edge profiles as input, showed that peeling-ballooning modes become more stable and more localised to the edge region (the radial eigenfunction of the unstable mode becomes narrower) with increasing triangularity and edge safety factor. A similar effect was observed when approaching closeness to double null. This narrowing, combined with the observation that access to second stability of ideal ballooning modes is closed when the density is increased, was proposed as an explanation for the reduced ELM size in type-II ELM regimes. According to this model, type-II ELMs should be narrower (smaller) but otherwise similar to type-I ELMs. We observe that the heat deposition pattern associated with the small bursts bears similarities with the type-I ELM heat deposition (in particular their propagation towards the inner divertor), but it is not immediately clear why magnetic precursor activity is observed for the small bursts and not for type-I ELMs (at least under co-injection). According to another model put forward by Lönnroth et al [31], the ELM type is determined by whether the edge transport barrier (ETB) is in the first or the second stability region for ideal ballooning modes. Type-I ELMs occur if the ETB enters the second stability region, while type-III ELMs occur if the stability of the whole ETB is determined by the first stability limit. Type-II ELMs occur if the first stability limit applies only to the outermost edge of the ETB. Hence, the distinction between type-II and type-III ELMs is related to the radial depth of the region unstable to ideal ballooning modes with $n=\infty$. According to this model, from the physics origin type-II and type-III ELMs should be very similar. Indeed, in similitude to type-III ELM observations, we also observe magnetic precursor activity prior to the type-II ELMs. However, this model does not explain why the typeIII ELM losses go preferentially to the outer divertor, while the losses associated with the small bursts are seen to go preferentially to the inner divertor.

It is important to note that, even if this regime becomes accessible in ITER, the presence of transient heat loads associated with the newly found small bursts may have consequences for the compatibility of the regime with ITER requirements. Recent material studies suggest an upper limit for the deposited energies of $0.5 \mathrm{MJ} / \mathrm{m}^{2}$ in order to avoid excessive target erosion (ultimately caused by anisotropy effects (CFC) or thermal fatigue (tungsten)) [32], and this is likely to lead to more severe limitations on the energy loss per ELM. Whether the small bursts in type-II ELM regime are indeed small enough for ITER will necessitate a more detailed study of the bursts, including additional aspects such as broadening of the power deposition profile or the temporal 

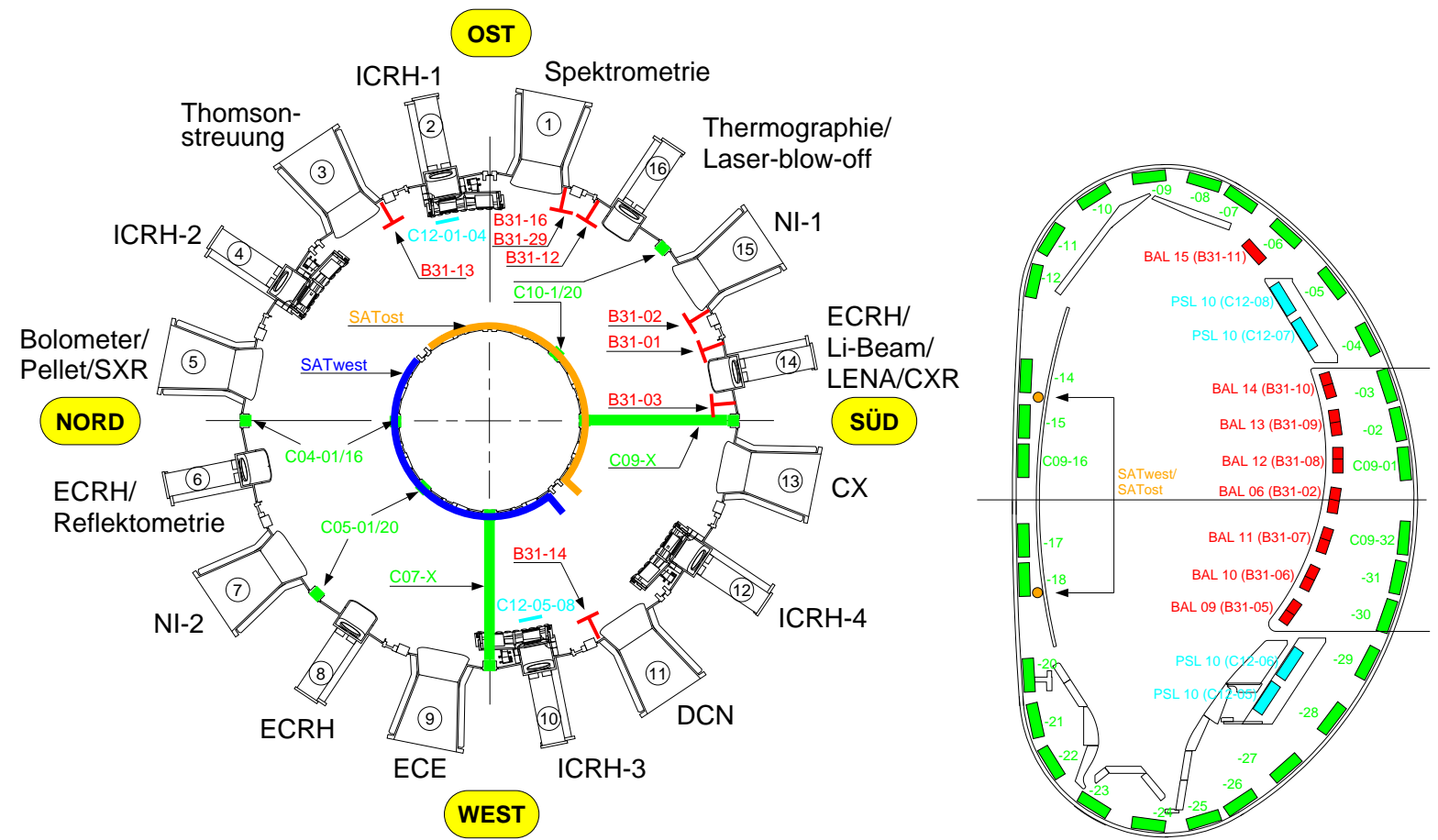

Figure A1. Toroidal (left) and poloidal (right) distribution of magnetic pick-up coils on ASDEX Upgrade.

shape of fluxes. This is left for future work.

\section{Acknowledgments}

This work was funded in part by the United Kingdom Engineering and Physical Sciences Research Council, by the European Communities under the contract of Association between EURATOM and UKAEA, and by a EURATOM Intra-European Fellowship. The views and opinions expressed herein do not necessarily reflect those of the European Commission.

\section{Appendix A. Additional information on the magnetic fluctuation diagnostic}

Figure A1 illustrates the spatial distribution of coils for magnetic fluctuation measurements, valid for the range of shots studied here. Two types of coils are $\underline{\text { available (see [33] for a more detailed description). The "C"-coils (marked in green) are }}$ $\underline{\text { conventional Mirnov coils (eff. surface }=0.36 \mathrm{~m}^{2} \text { ) measuring variations in the poloidal }}$ magnetic field. Amongst them there are two sets of C-coil arrays at different toroidal locations (C09-X, C07-X) covering the full poloidal cross-section. Of particular interest in this context is coil C09-16 (figures 6, 7), which is on the high field side and closest to the plasma boundary $(\sim 15 \mathrm{~cm}$ distance to the separatrix in our case). Note that the $\underline{\text { C-coils on the low field side are much further away from the plasma and have not been of }}$ $\underline{\text { much use for our purposes. The C-coil signals were acquired at variable sampling rates }}$ 
$\leq 250 \mathrm{kHz}$. The "B"-coils (marked in red) are printed circuit coils (eff. surface $=0.12$ $m^{2}$ ) that measure perturbations of the radial magnetic field. All B-coils are on the low field side. Compared to the C-coils on the outboard side, they are much closer to the plasma and hence more sensitive. A set of six toroidally distributed B-coils (mounted at the same poloidal angle) consisting of B31-14, B31-03, B31-01, B31-02, B31-12, and $\underline{B} 31-13$, is used for the identification of toroidal mode numbers (in figure 5, B31-01 is not used). For the shapes under consideration their distance to the separatrix is $\sim 14 \mathrm{~cm}$. The signals have a constant sampling rate of $500 \mathrm{kHz}$. To study even higher frequencies, a separate coil set is available (to which B31-16 in figure A1 belongs). Those signals are sampled with $2.5 \mathrm{MHz}$ and band-pass filtered $(0.1-1.0 \mathrm{MHz})$.

\section{References}

[1] Stober J et al 2001 Nucl. Fusion 411123

[2] Stober J et al 2005 Nucl. Fusion 451213

[3] Ozeki T et al 1990 Nucl. Fusion 301425

[4] DIII-D Team 1990 Plasma Phys. Control. Nucl. Fusion Res. 169 IAEA-CN-53/A-I-4

[5] Saibene G et al 2002 Plasma Phys. Control. Fusion 441769

[6] Saibene G et al 2005 Nucl. Fusion 45297

[7] Nunes I et al 2007 Proc. 34th EPS Conf. on Controlled Fusion and Plasma Physics (Warsaw) P5.137

[8] Silva A et al 1999 Rev. Sci. Instrum. 701072

[9] Sips A C C et al 2002 Plasma Phys. Control. Fusion 44 A151

[10] Kamada Y et al 1996 Plasma Phys. Control. Fusion 381387

[11] Horton L D et al 2004 Max-Planck-Institut für Plasmaphysik, private communication

[12] Perez C P et al 2004 Plasma Phys. Control. Fusion 4661

[13] Smeulders P et al 1999 Plasma Phys. Control. Fusion 411303

[14] Neuhauser J et al "Structure and Dynamics of Spontaneous and Induced ELMs on ASDEX Upgrade", presented on 21st IAEA Fusion Energy Conference (2006), Chengdu (China), IAEA$\mathrm{CN}-149 / \mathrm{EX} / \mathrm{P} 8-2$.

[15] Neuhauser J et al, submitted to Nucl. Fusion

[16] da Graça S et al, to be presented at 35th EPS Conf. on Controlled Fusion and Plasma Physics (Crete, 2008)

[17] Mc Carthy P J 1999 Phys. Plasmas 63554

[18] Herrmann A et al 1995 Plasma Phys. Control. Fusion 3717

[19] Herrmann A 2007 Phys. Scr. T128 234

[20] Herrmann A 2001 Proc. 28th EPS Conf. on Controlled Fusion and Plasma Physics (Madeira) P5.104

[21] Fuchs J C et al 2001 J. Nucl. Mater. 290-293 525

[22] Eich T et al 2005 Plasma Phys. Control. Fusion 47815

[23] Eich T et al 2007 J. Nucl. Mater., doi:10.1016/j.jnucmat.2007.01.240 (in press)

[24] Suttrop W et al 1996 Plasma Phys. Control. Fusion 381407

[25] Kass T et al 1997 Proc. 24th EPS Conf. on Controlled Fusion and Plasma Physics (Berchtesgaden), vol. 21A, ed M. Schittenhelm et al (Geneva: EPS) part IV, p. 1521

[26] Cupido L, da Graça S, Conway G D, Manso M, and Serra F 2006 Rev. Sci. Instrum. 77 10E915

[27] da Graça S et al 2007 Plasma Phys. Control. Fusion 491849

[28] Perez von Thun C P et al 2007 Proc. 34th EPS Conf. on Controlled Fusion and Plasma Physics (Warsaw) D5.006

[29] Mazurenko A et al 2002 Phys. Rev. Lett. 89225004 
[30] Saarelma S, Günter S, Horton L D and ASDEX Upgrade Team 2003 Nucl. Fusion 43262

[31] Lönnroth J-S et al 2003 Plasma Phys. Control. Fusion 451689

[32] Zhitlukhin A et al 2007 J. Nucl. Mater. 363-365 301

[33] Gernhardt J 1992 "Magnetic diagnostic on ASDEX Upgrade with internal and external pick-up coils", IPP-Report $1 / 262$ 